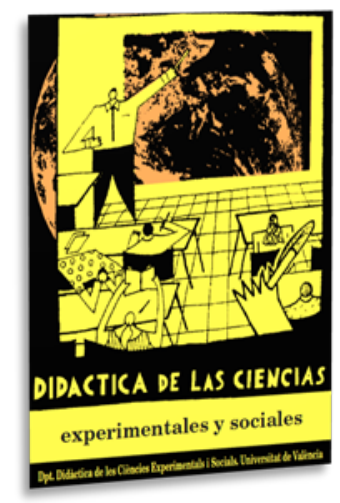

\title{
La empatía histórica, una propuesta didáctica clave para la Formación Profesional Básica
}

\author{
Historical empathy, a key teaching proposal \\ for Basic Vocational Education and Training
}

DOI: $10.7203 / D C E S .40 .17309$

\author{
Carlos D. Ciriza-Mendívil \\ Universidad Pública de Navarra, carlos.ciriza@unavarra.es \\ ORCID iD: http://orcid.org/0000-0002-2193-5978
}

RESUMEN: La adquisición del pensamiento histórico, así como el desarrollo de las competencias históricas son objetivos fundamentales de la educación de esta disciplina. Y, sin embargo, su desarrollo y consecución en el aula de Formación Profesional Básica (FPB) conlleva enormes complejidades. Este artículo presenta un análisis de caso en el que se emplea una metodología de corte cualitativo, basada en la investigación-acción a partir de una propuesta didáctica en el módulo de Comunicación y Sociedad de la FPB analizada mediante dos tipologías instrumentales, una plantilla de observación y diez entrevistas. En la línea con otros análisis de las competencias históricas y vinculándolos con estudios propios de la FPB este análisis defiende la empatía histórica, indirectamente relacionada con la empatía cultural, la vinculación de la historia a los contextos de los estudiantes y la interdisciplinaridad como los medios para el desarrollo del pensamiento histórico y las competencias históricas en el alumnado de FPB.

PAlABRAs ClAVE: empatía histórica, Formación Profesional Básica (FPB), pensamiento histórico, propuesta didáctica, didáctica de las ciencias sociales

ABstract: One of the main goals of teaching history has been, for the last decades, historical thinking, as well as the development of historical competences. However, the development and achievement of this goal in Basic Vocational Education and Training (VET) involves enormous complexities. This article presents a case study in which a qualitative methodology based on action research is used through a teaching proposal for the Communication and Society module. Its findings have been collected using two main tools, a sheet for observation and ten interviews. Following some researches about historical thinking, this article supports the idea that historical empathy, indirectly related with cultural empathy, linking history to the students' contexts and interdisciplinarity in our ways of teaching are the main tools and the best way for developing full historical thinking in the students of basic vocational education and training.

KEYWORDS: historical empathy, Basic Vocational Education and Training (VET), historical thinking, teaching proposal, social sciences teaching

Fecha de recepción: mayo de 2020

Fecha de aceptación: junio de 2020

Mis agradecimientos a la Dra. Ana Mendioroz Lacambra por su ayuda y consejo en la elaboración y redacción de este artículo. 


\section{INTRODUCCIÓN}

Con la entrada en vigor de la Ley Orgánica de la Mejora de la Calidad Educativa (LOMCE) en 2014 numerosos elementos, cursos y planes educativos se vieron modificados. Uno de ellos, quizás uno de los más evidentes, fue la introducción de la Formación Profesional Básica (FPB) que pasaba a sustituir a los Programas de Cualificación Profesional Inicial (PCPI). Desde antes de sus inicios, los debates y problemáticas en torno a esta nueva formación han sido constantes. Más aún, ahora que se vislumbra la llegada de un nuevo anteproyecto de ley con la Ley Orgánica de Modificación de la Ley Orgánica de la Educación (LOMLOE), las incertidumbres en torno a su continuidad y a su desempeño reaparecen con especial presencia.

Y, sin embargo, el riesgo de presentar modificaciones a esta formación sin la debida profundización, o incluso sin haber permitido a las distintas implementaciones educativas desarrollarse en el tiempo (García Gómez, 2014) parece muy real. Así pues, en este momento más que nunca, los proyectos educativos innovadores ${ }^{1}$ y la investigación sobre su práctica en el aula, sus limitaciones y posibilidades, son cada vez más necesarios. Al fin y al cabo, solo de esta manera podremos enfrentar algunos de los principales problemas que afectan a la FPB y a su alumnado.

Este estudio presenta una propuesta didáctica en el que investigador, docente y práctica en el aula se unen, partiendo de un problema inicial generalizado, esto es, la falta de interés en la historia por parte del alumnado de FPB. Para afrontar dicha problemática, se plantea como hipótesis y objetivo principal el desarrollo de la empatía histórica para construir un pensamiento histórico crítico-creativo (Santisteban Fernández, 2010, p. 39) y desarrollar las competencias históricas (Seixas y Morton, 2013).

Esta propuesta, partiendo de este objetivo principal, desarrolla dos objetivos específicos. De un lado, desarrollar la vinculación entre la disciplina histórica y las vivencias cercanas de los alumnos y de sus familias (Almansa Pérez, 2018, p. 17). Así, utilizar la dimensión ética, la motivación por lo cercano, por "sus propios contextos e intereses” (Rivero y Pelegrín, 2015, p. 20) y por los agentes históricos conocidos como factor motivante del alumnado. Y de otro lado, fomentar las propuestas didácticas interdisciplinares que vinculan la disciplina histórica con la lingüística. Esta interdisciplinariedad, que es una necesidad curricular en los módulos comunes de la FPB, se desarrolla a través del uso de fuentes históricas de variada tipología, especialmente, orales (Burke, 2005; Leoné Puncel y Mendiola Gonzalo, 2007).

\section{MARCO TEÓRICO}

\subsection{La FPB y sus especificidades}

La Formación Profesional Básica, si bien fue una de las incorporaciones de la LOMCE, puede vincularse a programas relativamente similares cuya andadura comenzó con la LOGSE y llegó hasta nuestros días a través de la LOE y los PCPI (Cutanda López, 2014, p. 70). A rasgos generales, la FPB -que iniciaba su andadura en el curso escolar 2014-2015- quedaba organizada en dos cursos $\mathrm{y}$, aunque con leves diferencias entre comunidades, a ella podían acceder los alumnos de los 15 a los 17 años, siempre que hubieran cursado el primer ciclo de secundaria o, al menos, el segundo curso de la misma, y fueran propuestos para su incorporación a este ciclo por el profesorado de su centro de origen (LOMCE, 2013, art. 41.1). Planteada como una "segunda oportunidad" (Cutanda López, 2014, p. 70), pero también como un medio para reducir el abandono escolar prematuro y las situaciones de riesgo social de un determinado alumnado (Alemán Falcón, Calcines Piñero y Santana Hernández, 2017); la compleja evaluación final que debe superar el discente, cuestiona a la

\footnotetext{
${ }^{1}$ Véase, como ejemplos, para distintos ciclos educativos: Moreno, España y Blanco, 2016; Martín Ayasta, 2010; Perarnau y Vieyra, 2017; Ríos Rincón, 2017; Molina Puche y Egea Zapata, 2018; Rivero y Pelegrín, 2015.
} 
luz de los análisis de Fernández-García, García Llamas, y García Pérez (2019, p. 226) su papel como "segunda oportunidad”.

En cualquier caso, a los efectos de esta propuesta, cabe señalar que la principal problemática de este alumnado no reside en las asignaturas profesionalizantes (Bello Muñoz y Carabantes de las Heras, 2015, p. 69), sino en los módulos comunes y obligatorios de la FPB -Comunicación y Ciencias Sociales por un lado, y Ciencias Aplicadas por otro- que concentran las asignaturas de Matemáticas, Ciencias Naturales, Lengua Castellana, Lengua extranjera, Ciencias Sociales y lenguas cooficiales de cada comunidad autónoma (LOMCE, 2013, art. 42, p. 33). Paradójicamente, es en estos módulos y asignaturas comunes en los que reside, de una parte, la base competencial para poder obtener el graduado de ESO y, de otra parte, los elementos generales que, como la empatía o la comunicación, pueden tener una gran proyección en la vida cotidiana futura de este alumnado (Cid-Sillero, Santiago-Ramajo, y Martín-Lobo, 2018, p. 520).

Sin embargo, las dificultades en los procesos de enseñanza-aprendizaje del módulo de Comunicación y Ciencias Sociales son amplias, lo que, en consecuencia, limita las posibilidades de un verdadero desarrollo del pensamiento histórico. En primer lugar, el desarrollo excesivo y casi inabarcable del currículum de estas disciplinas que, en muchos casos, es la mera adaptación resumida del currículum de la ESO. Así, para algunos autores, se configuraron unos módulos comunes de comunicación y sociedad que, paradójicamente, ni comunican ni fomentan la aplicación de las aptitudes sociales y de sus competencias (Bello Muñoz y Carabantes de las Heras, 2015, p. 70). En segundo lugar, la escasa adaptación y contextualización de estas materias comunes. Si bien, en teoría, las mismas debían vincularse "al campo profesional del perfil del título” (Cejudo Cortés y Corchuelo Fernández, 2018, p. 64), no es habitual que así sea. Al mismo tiempo, el propio currículum limita y relega en gran medida las adaptaciones curriculares que sí se desarrollaban con los PCPI. Y en tercer lugar, el hecho de que el profesorado se enfrenta a un alumnado que ha fracasado previamente en esta diciplina, por lo tanto, sumamente desmotivado (Fernández-García, García Llamas y García Pérez, 2019, p. 215) y con unos conocimientos previos cuya limitaciones nada tienen que ver con los objetivos y exigencias del currículum de la FPB.

\subsection{La empatía histórica}

La empatía como uno de los elementos relevantes para desarrollar competencias históricas en el aula, tiene una amplia literatura (Trepat, 1995; González, Henríquez, Pagès y Santiesteban, 2009; Martín Ayasta, 2010; Domínguez, 2015; Rivero y Pelegrín, 2015; Perarnau y Vieyra, 2017; Ríos Rincón, 2017; Salmón Perrilliat, 2017; Almansa Pérez, 2018; Molina Puche y Egea Zapata, 2018). Al fin y al cabo, como señalara Davis (1983) la empatía es un constructo multidimensional con numerosos elementos afectivos cognitivos, contextuales (Butrus y Witenberg, 2013, p. 292), lo que ha hecho que sea un elemento de análisis en numerosos ámbitos y disciplinas. En términos generales, podemos definir la empatía (Perarnau y Vieyra, 2017, p. 3) como el concepto que "hace referencia a ponerse en situación de los otros” y, al mismo tiempo, hacerlo "mirando las cosas desde su punto de vista”. O lo que es lo mismo, "el reconocimiento de que cualquier grupo social, incluido el nuestro, resulta totalmente exótico si se mira de lejos y en consecuencia pierde ese exotismo con la cercanía” (Salmón Perrilliat, 2017, p. 377).

Teniendo en cuenta la importancia de este constructo en el desarrollo moral adolescente, en su educación cultural y en su creación de vínculos sociales (Eisenberg, 2000), no sorprende su pronta vinculación con la didáctica de las ciencias sociales. Así, su implementación y desarrollo, muy vinculada a la adquisición del pensamiento histórico como objetivo de la enseñanza de la historia (Santisteban Fernández, 2010, p. 47), es habitual, al menos, desde la última década del siglo XX (Almansa Pérez, 2018, p. 91) En todo caso, pese a que los estudios, por ejemplo, para el caso de España, abordan la empatía histórica en la educación primaria, la educación secundaria e incluso en la formación del futuro profesorado de ambas etapas educativas, siguen siendo escasos en las 
formaciones profesionales medias y superiores y, prácticamente inexistentes, en la formación profesional básica. Y, sin embargo, más allá del ciclo educativo en el que vaya a implementarse, la empatía es parte fundamental y esencial del desarrollo del pensamiento histórico, más aún si observamos el mismo desde una dimensión ética (Molina Puche y Egea Zapata, 2018, p. 4).

En cualquier caso, la empatía histórica no puede tomarse como un constructo unitario en el que su consecución sea total o nula. Por el contrario, se trata de una parte del pensamiento histórico cuyo desarrollo ya fue dividido en niveles por Shemilt (1984). Así, aunque más adelante esta división fuera revisada por numerosos autores (Trepat, 1995; Díaz Barriga, 1998; González, Henríquez, Pagès, y Santiesteban, 2009; Lee y Shemilt, 2009) dando lugar a distintas escalas, todas ellas, en definitiva, comienzan con un primer estadio en el que se percibe a los antepasados como individuos menos desarrollados y menos conocedores de su contexto que las personas del presente. A continuación, se sucederían una serie de estadios cuyo número varía, pero que comenzarían a considerar a los individuos del pasado como seres humanos iguales a los individuos de la actualidad, y cuyo conocimiento parte de la profundización en las fuentes primarias. Y una última fase -cuarta o quinta en función de los distintos investigadores- en la que la empatía histórica ha sido desarrollada por completo en el aula. Así, es observable en las redacciones y dinámicas de los alumnos, los cuales consideran a los individuos del pasado como seres humanos tan capaces como los individuos del presente, pero cuyas formas de pensar y percibir el mundo varían, no en función de su capacidad, sino del contexto que les rodea.

Más allá de los debates pedagógicos en lo relativo a la gradación y escalas de consecución de la empatía histórica, es bien probable que su principal problemática no haya sido eso, sino la implementación de este concepto en el aula y su consecuente evaluación. Así, se puede desarrollar la combinación de instrumentos, técnicas y herramientas de evaluación competencial variadas (Molina Puche y Egea Zapata, 2018), esperando que, de esta manera, el desarrollo y su evaluación sean exitosos. Sin embargo, las opciones para la implementación didáctica de la empatía histórica siguen siendo limitadas. Además, presentan numerosas complicaciones como las referidas por dichos autores, pero también observadas en otros análisis como los de Cid-Sillero, SantiagoRamajo, y Martín-Lobo (2018).

A pesar de ello, la empatía histórica y la dimensión emocional otorgan al proceso de enseñanza-aprendizaje numerosas posibilidades (Rivero y Pelegrín, 2015, p. 21). Desde su consecuente impacto en las relaciones sociales del alumnado (Salguero, Fernández-Berrocal, RuizAranda, Castillo, y Palomera, 2011), hasta las reacciones y disposiciones positivas ante las propias disciplinas humanísticas (Almansa Pérez, 2018), pasando por la vinculación del pasado con los problemas actuales tanto de la sociedad como del propio alumnado (Fernández-García, García Llamas, y García Pérez, 2019, p. 215).

En consecuencia, uniendo la dimensión ética del pensamiento histórico, con la empatía histórica, y ésta con la vinculación individual del alumnado con el pasado, esta propuesta didáctica abandona los análisis más tradicionales, memorísticos y casuísticos -que todavía están muy presentes en la enseñanza de la historia (Doñate Campos y Ferrete Sarria, 2019, p. 48)- para pasar a abordar las competencias, en este caso históricas, que buscan el desarrollo procedimental de esta disciplina desde la acción, las estrategias didácticas y el rol activo del discente.

\section{MÉTODO}

Esta propuesta presenta una investigación de tipo cualitativo circunscrita a un planteamiento propio de la investigación-acción en educación. A fin de cuentas, como señalara Latorre (2003, págs. 25-27) este planteamiento metodológico se caracteriza, entre otros elementos, por ser cíclico, participativo, reflexivo y eminentemente cualitativo, lo que caracteriza la presente propuesta, así como sus herramientas y técnicas de recolección de datos. Así, se trata aquí de conectar dos mundos 
en ocasiones alejados; de un lado, los avances teóricos en el campo educativo y, de otro lado, la práctica cotidiana de las aulas y los centros (Ortiz y Borjas, 2008, p. 620).

Más aún, siguiendo la clasificación de Carr y Kemmis (1988) esta propuesta se incluiría dentro de la investigación-acción técnica, esto es, una investigación cuyos objetivos buscan la efectividad y eficiencia, así como la mejora de la práctica y el desarrollo docente, siendo el investigador un elemento externo, pero cercano a la práctica diaria del aula y a la propuesta didáctica. Esta tipología, si bien requiere de una cooperación muy amplia entre el docente y el investigador, también permite valorar los resultados desde un punto de vista algo más alejado de la práctica docente, incluyendo al propio profesorado entre los informantes de la investigación, lo que nos permitía desarrollar esta propuesta y analizar al máximo sus resultados.

Se invita a participar en esta propuesta a varios centros de formación profesional adscritos al departamento de educación de Navarra. Finalmente, se selecciona el Centro de la Fundación Laboral de la Construcción en Villafranca (Navarra) en el que se imparte Formación Profesional Básica de Fabricación y Montaje (FPBFyM). Este centro concertado cumple con varias de las características que se consideraban esenciales para desarrollar esta propuesta. En primer lugar, se trataba de un centro educativo diferente a las instituciones educativas en las que el alumnado había desarrollado sus trayectorias educativas previas, lo que permitía fomentar un nuevo contexto educativo (González González y Moreno Yus, 2013, p. 232). En segundo lugar, se trata de un centro de una trayectoria que comenzó con los PCPI, lo que permitía contar con un profesorado que, para los módulos comunes, contaba con experiencia en el centro y con el tipo de alumnado. Y en tercer lugar, se trata de un centro concertado que imparte únicamente esta formación profesional y que cuenta con un número reducido de alumnos, lo que permitía analizar en profundidad los resultados a un número total de participantes que asciende a 12 estudiantes y una profesora.

El análisis de los resultados de la propuesta se llevó a cabo a través de 13 entrevistas. Se trata de entrevistas semiestructuradas tanto al alumnado como a la profesora que llevó a cabo la propuesta. Para la selección temática de las cuestiones a plantear en estas entrevistas, las mismas siguieron trabajos previos de validación de escalas sobre la empatía (González-González, ÁlvarezCastillo, y Fernández-Caminero, 2015). Al mismo tiempo, estas herramientas de obtención de información se elaboraron también fundamentándose en las mediciones de gradaciones emocionales durante los proyectos desarrolladas por Moneo-Marín, Jiménez-Pérez, y Jiménez-Palacios (2017, págs. 75-77). Por su parte, la profesora, guiada por una plantilla de observación, recogió en las sesiones de esta propuesta distintas cuestiones tales como la participación del alumnado, su interés, las preguntas y dificultades encontradas, la eficacia de las actividades desarrolladas, así como la detección de rasgos propios de los distintos niveles de empatía histórica (Shemilt, 1984; Trepat, 1995; Díaz Barriga, 1998; González, Henríquez, Pagès, y Santiesteban, 2009, Lee y Shemilt, 2009; Lee y Shemilt, 2011).

Esta propuesta se enmarca en la asignatura de Comunicación y Sociedad del primer curso de FPBFyM que, para el caso de la Comunidad Foral de Navarra y según el Decreto Foral 73/2014 de 27 de agosto, se divide en unidades formativas las cuales "integran conocimientos básicos relativos a ciencias sociales, lengua castellana y, en su caso, lengua vasca, y literatura y lengua inglesa”. En consecuencia, toda propuesta didáctica -más aún si se trata de un proyecto de investigación-acción que parte de una situación real- debe ser marcadamente interdisciplinar. De lo contrario, dados los tiempos y cronogramas educativos, sería completamente ineficaz en la práctica del aula, perpetuando el ya señalado “divorcio” entre investigación y docencia (Oliva Martínez, 2012).

Así pues, el presente proyecto, diseñado para una duración total de 3 meses, desarrolla los apartados propios de la unidad formativa 1 y combina las disciplinas de historia y lengua castellana con los siguientes resultados de aprendizaje, criterios de evaluación y contenidos.

Esta propuesta didáctica se construye en tres fases:

Fase de motivación. La primera etapa del proyecto, establece los planteamientos generales a evaluar, los conocimientos previos, así como el contexto motivacional del alumnado hacia el 
proyecto. De una parte, se desarrolla "una estrategia comparativa como modo de comprensión de problemática comunes” que se ha probado exitosa en otros contextos (Almansa Pérez, 2018, p. 95). De otra parte, se presenta el objetivo final del proyecto, esa creación documental con la que además de acercar la historia a sus vidas cotidianas se trataba de motivar al alumno con un objetivo educativo distinto, participativo y competencial. Finalmente, se evalúan los conocimientos previos del alumnado tanto en el aspecto histórico como en el del lenguaje.

Fase de desarrollo. Para esta segunda fase se cuenta con dos ámbitos o espacios diferenciados, esto es, dentro y fuera del contexto del centro escolar. En el primer ámbito, se desarrollan las directrices generales necesarias para una entrevista. El grupo se divide en parejas y establece, a partir de las ideas generales observadas, del trabajo en el aula y de sus conocimientos previos, los temas a tratar. En este caso, se estableció que la entrevista sería a mujeres de sus familias y los temas serían: trabajo, familia, derechos, vivienda y sociedad. Para desarrollar estas temáticas se organizó el aula en diversas sesiones en las que el grupo se dividió en grupos mayores organizados a partir de la metodología "grupo de expertos" Una vez desarrolladas las temáticas en el aula a partir de la investigación en estos grupos, quedan las cuestiones metodológicas sobre la fuente, es decir, las entrevistas. Tras el visionado y lectura de diversas entrevistas en las que se comparan entrevistas científicas, periodísticas, radiofónicas o televisivas se desarrolla, mediante el debate en el aula una entrevista semiestructura modelo para que los alumnos la implementaran en sus casas. En el segundo ámbito fuera del contexto escolar, el trabajo se divide por parejas. Individualmente los alumnos llevan a cabo la entrevista, grabándola con sus dispositivos móviles. Tras esta entrevista, analizan las respuestas y, a partir de los temas tratados, resumen las ideas planteadas por la entrevistada. Finalmente, antes de volver al aula, resta la parte de análisis y comparación que, para este caso, y dada la dispersión geográfica del alumnado -que se especificará después-, se hace por parejas fuera del centro y, por grupos a través de la metodología "lápices al centro" de vuelta en el aula. Se trata así de que el alumnado, además de trabajar en grupo, tome decisiones trascendentales sobre su propio trabajo, una competencia específica de suma importancia tanto en el proceso educativo como en el futuro de los alumnos (Montserrat y Melendro, 2017).

TABLA 1 Guion modelo de las entrevistas elaboradas por los alumnos

\section{Introducción a la entrevista}

Saludo y presentación del entrevistado.

¿Cuándo nació? ¿Dónde nació?

¿Cómo era el pueblo grande o pequeño? ¿ ¿

\section{Desarrollo de la entrevista}

¿Cómo era la casa en la que vivías? ¿Cuántos vivíais en la casa? ¿Había cuarto de baño, agua corriente, luz...?

¿A qué años te casaste? ¿Te mudaste entonces?

¿Cuál era el trabajo de tus padres? ¿En la casa quién hacía las cosas? ¿Cómo ibas a trabajar? ¿Qué ropa de trabajo utilizabas?

¿Había colegio donde vivías? ¿Cómo eran las clases? ¿Cuántos maestros había?

¿Había médico en el pueblo? ¿El médico y la escuela eran gratis? ¿Qué tiendas había? ¿Había bares? ¿Quién entraba en los bares?

¿A qué jugabais? ¿Salías todos los días a la calle? ¿Qué se hacía los domingos? ¿Cómo eran los juegos?

\section{Conclusión y cierre de la entrevista}

¿Cuál es la mayor diferencia de tu infancia con la actualidad? ¿Lo mejor? ¿Y lo peor?

* Subrayadas las preguntas incorporadas por los estudiantes en clase 
Fase de discusión final. Con la intención de que la práctica diaria en el aula se beneficie al máximo posible de una motivación del alumnado difícil de conseguir, esta última fase se prolonga y divide en tres grandes etapas. Una primera en la que se desarrollan distintas presentaciones que comparan los resultados de las distintas entrevistas de los miembros del grupo. Siguiendo las temáticas principales, se trata de construir un relato grupal que muestre las distintas variedades. Una segunda de debate y discusión en la que, de forma grupal, se presentan ante el aula los distintos relatos y se promueve la discusión y la comunicación de resultados en público. Se fomenta así tanto la comunicación, como la empatía efectiva hacia las visiones y opiniones del otro en un contexto comunicativo informal. Y una tercera en la que se construye un relato histórico común del aula. De esta manera, se fomenta un aprendizaje participativo y democrático en el que el grupo, empatizando con las distintas realidades, pero también con las distintas percepciones de sus compañeros de clase, desarrolla una visión de conjunto en la que se incluyen las distintas realidades generacionales, de época y de lugar.

\section{RESULTADOS Y DISCUSIÓN}

Se presentan algunas de las evidencias obtenidas a lo largo de las distintas fases de esta propuesta, especialmente, a partir de la plantilla de observación de la profesora y las entrevistas llevadas a cabo una vez finalizada la propuesta didáctica. Dado que las directrices principales de esta entrevista, su temática y su estructura ya han sido descritas y, por lo tanto, puede ser replicada y adaptada a otros análisis, la misma no se incluye en este estudio. En todo caso, sí lo hacen algunos de los códigos y categorizaciones mediante los cuales se ha implementado el análisis cualitativo de este estudio.

El inicio de las entrevistas -tanto a la profesora como al alumnado- se vincula al objetivo principal de esta propuesta, esto es, el desarrollo de la empatía histórica para fomentar el pensamiento histórico y sus competencias. Para ello, la codificación "empatía” y su "dominio cultural” se analiza a través de redes semánticas, KWIC y comparaciones pareadas (Ryan y Bernard, 2003).

TABLA 2 Fases de la empatía (Lee y Shemilt, 2009; 2011) y su codificación para el análisis de las entrevistas

\begin{tabular}{lllll}
\hline Fases de la empatía & \multicolumn{1}{c}{ Elemento esencial } & \multicolumn{2}{c}{ Codificación/Subtemas } \\
clave
\end{tabular}

Fuente: Elaboración propia 
En lo que respecta a la profesora, la misma constató a través de la plantilla de observación desarrollada que, a pesar de las grandes deficiencias en los conocimientos previos mostradas por algunos alumnos tales como el sujeto 10 que, según la profesora "no había hecho un examen desde primaria", se produjo un gran avance a lo largo de la propuesta. Como señalara la docente en su entrevista, "ahora los alumnos ven la historia a su alrededor, no lo mencionan, pero creo que son conscientes que cuando hablamos de historia también hablamos de sus propias vidas y eso ha tenido mucha importancia”. Así, el desarrollo del pensamiento histórico a través de este aprendizajesignificativo permite, en palabras de la profesora, "alcanzar cuestiones y tratar temas que aparecen en el libro y en el currículum pero que casi nunca se pueden llegar a impartir porque son muy extensos”.

En lo que respecta al reflejo del objetivo principal en el caso de las entrevistas a los alumnos, se observa el desarrollo de la empatía histórica, aunque a diferentes niveles (Shemilt, 1984; Trepat, 1995; Díaz Barriga, 1998; González, Henríquez, Pagès, y Santiesteban, 2009). Desde aquellos sujetos que consideran a los antepasados como individuos similares a los del presente con afirmaciones tales como las del sujeto 1: "me he dado cuenta que al final todos somos parecidos" o el sujeto 7: "en mi familia mi abuela y yo hemos pasado por las mismas cosas y nos parecemos porque yo hubiera hecho lo mismo que hizo ella”; hasta, las últimas fases de la empatía histórica en la que se considera a los agentes históricos como individuos totalmente capaces, pero con formas de ver y pensar distintas a las actuales (sujetos 2,3,5, 8 y 9). Cabe señalar que el código/dominio cultural más observado en las entrevistas, si bien se vincula con la empatía histórica, muestra sus fases intermedias, este es, la humanización de los agentes históricos.

TABLA 3 Fases de la empatía (Lee y Shemilt, 2009; 2011) alcanzadas por los sujetos analizados y elementos de la codificación predominantes en sus entrevistas

\begin{tabular}{|c|c|c|}
\hline $\begin{array}{l}\text { Sujetos del estudio de } \\
\text { caso }\end{array}$ & $\begin{array}{l}\text { Fase de la empatía } \\
\text { alcanzada }\end{array}$ & $\begin{array}{l}\text { Correspondencia con } \\
\text { categorizaciones de tabla } 1\end{array}$ \\
\hline Sujeto 1 & III nivel & PR/EF/LP \\
\hline Sujeto 2 & V-VI nivel & AH/FH/EH/EC \\
\hline Sujeto 3 & IV nivel & ER/LP/AH/CC \\
\hline Sujeto 4 & III nivel & VA/ER/D/AD \\
\hline Sujeto 5 & IV nivel & ER/AH/CC \\
\hline Sujeto 6 & I nivel & NE/PR \\
\hline Sujeto 7 & III nivel & $\mathrm{ER} / \mathrm{D} / \mathrm{AD}$ \\
\hline Sujeto 8 & V-VI nivel & AH/CC/FH/EH/EC \\
\hline Sujeto 9 & V nivel & $\mathrm{AH} / \mathrm{FH} / \mathrm{EH}$ \\
\hline Sujeto 10 & I nivel & NE/PR \\
\hline
\end{tabular}

Más aún, las entrevistas al alumnado también muestran la aparición de un resultado indirecto que, si bien forma parte de los objetivos de la FPB señalados, no formaba parte de los objetivos iniciales de este proyecto, a saber, la relación entre la empatía histórica y la empatía cultural (González-González, Álvarez-Castillo, y Fernández-Caminero, 2015). Esto es, la vinculación que el grupo de alumnos hace entre pasado-presente, pero también entre el pasado de los distintos lugares y el presente de los distintos lugares para favorecer ese ejercicio de ponerse en el lugar del otro, tanto en el pasado, como en el momento actual. No sorprende que esta relación se observe especialmente -aunque no siempre- en aquellos alumnos que alcanzan las últimas etapas de la 
empatía histórica (fundamentalmente sujetos 2,3,5 y 8). Dicha vinculación es constatable a través de afirmaciones como las del sujeto 8: "la abuela de [...] aunque era de Marruecos se divorció, pero en España eso no se podía, eso me ha llamado la atención, que en el pasado las cosas fueran también distintas, pero al revés"; o las del sujeto 2 que afirma "me llamó la atención que mi familia en Marruecos tenía una casa y la abuela de [...] vivía en una cueva en Arguedas (Navarra)”. Bien por la propuesta didáctica, por el enfoque del planteamiento o porque la empatía histórica tiende, como señalaran algunos estudios etnográficos a la empatía cultural, lo cierto es que los alumnos vinculan ambas, siendo en algunos casos indisociables en varias de sus respuestas que unen pasado con presente, pero también los conceptos del "yo" y "el otro".

La entrevista a la profesora, aunque remarca esta misma vinculación, también observa el relativo fracaso de esta propuesta didáctica para conseguir sus objetivos en algunos sujetos (especialmente sujetos 4,6, y 10). La escasa empatía histórica alcanzada por estos alumnos -que se corrobora en sus entrevistas- es, según la profesora, causada por la escasa utilidad del primer objetivo específico -la búsqueda de cercanía histórica para motivar al alumnado- en algunos sujetos. Según constata la docente "en algunos alumnos se puede ver que acercar la historia a sus familias les motiva, mejora su relación con sus madres y, sobre todo, con sus abuelas, que suelen generar emociones positivas. Pero, en otros alumnos, como por ejemplo [sujetos 6] y [sujeto 10] la cercanía no tuvo este efecto y su falta de interés por la asignatura continuó”.

Vinculado a este primer objetivo específico, se observa la importancia de dos cuestiones fundamentales en las entrevistas. De una parte, en aquellos sujetos en los que la propuesta tuvo menos éxito, este resultado puede vincularse, especialmente, a varias de las cuestiones observadas en otros estudios sobre este alumnado. A saber, la falta de madurez (Moneo-Marín, Jiménez-Pérez, y Jiménez-Palacios, 2017) señalada por la profesora, los altos índices de desmotivación y falta de confianza en torno al sistema educativo (Olmos Rueda y Mas Torelló, 2013) observados en las entrevistas a los alumnos y, especialmente, los problemas económicos y afectivos que se originan en las familias de las que provienen estos discentes (Rodríguez Sabiote, Álvarez Rodríguez, y Moreno Yus, 2009, p. 188). Sin embargo, casi paradójicamente, la disponibilidad y cercanía física de familiares, si bien en caso de darse era habitualmente positiva, en los casos en los que no existía no fue negativa. Así ocurrió con los sujetos 1 y 5, que llevaron a cabo sus entrevistas online con sus familiares en otros países en África y América Latina. Por el contrario, para otros alumnos como el sujeto 6 o el sujeto 10 la cercanía física no supuso un elemento positivo ni motivador. La plantilla de observación de la profesora constata su poca participación y desmotivación, la cual no es achacable a ninguna condición desfavorable objetiva y observable, problemática ya constatada para la FPB en otros estudios (Corbacho Casas y Pons García, 2006, p. 242).

De otra parte, los participantes muestran la importancia de un elemento sustancial de esta propuesta y que debe ser tenido en cuenta, la propia situación del centro educativo como una institución ajena y distinta a aquellas en las que desarrollaron sus trayectorias previas. Si bien, como evidencia algunas de las entrevistas el cambio de centro repercute negativamente en las relaciones de los alumnos, ya que muchos de ellos no se conocen previamente, el desarrollo de esta propuesta didáctica convierte dicha problemática en un elemento positivo (González González y Moreno Yus, 2013). Así, la profesora constata que "un problema inicial en estos centros es que muchos alumnos no se conocen, por lo que estas actividades han hecho que se conozcan algo más, tanto sus vidas como sus familias". En consecuencia, señala la docente que "el trabajo en grupo ha funcionado mejor que en otras ocasiones, probablemente porque se trataba de sus familias y los alumnos estaban más implicados" y en la plantilla de observación constata cómo "se puede observar la mejora en las relaciones dentro del aula, por ejemplo, en los trabajos en grupo de las actividades". De igual manera, las entrevistas a los alumnos evidencian estas situaciones tanto positivas como negativas. Así, como señala el sujeto 3 en cuanto a su implicación y trabajo en la propuesta "es la primera vez que hago tareas que nos han mandado para casa”. Al mismo tiempo, el sujeto 7 afirma en lo relativo a las relaciones sociales previas que "solo conocía antes al [sujeto 2] pero no estaba 
en mi grupo" y, poco después en lo que respecta al trabajo en grupo menciona que "ha estado bien porque todos hacíamos algo”.

TABLA 4. Guion de la entrevista semiestructurada a los alumnos

\title{
Preguntas de apertura/inicio
}

\begin{abstract}
A lo largo de las semanas hechos hecho un proyecto, ¿Qué te ha parecido? ¿Y las entrevistas? ¿Habías hecho antes?

¿Qué entrevista has realizado? ¿A quién?

¿Con qué compañero/a de clase la preparaste? ¿Os conocías de antes? ¿Qué tal el trabajo con tu compañero/a?

¿A quién se la hizo tu compañero? ¿Se parecían?
\end{abstract}

\section{Preguntas sensibles/específicas}

Sobre la entrevista, ¿Dónde la hiciste? ¿Estabais el entrevistado/a y tú solos? ¿Cuáles fueron los temas de los que hablasteis? ¿Y tu compañero de clase? ¿Qué te llamó la atención de tu entrevista?

¿Qué opinas de los lugares donde vivían antes? ¿Y de los trabajos?

¿Crees que la persona entrevista, por ser mujer, tuvo problemas? ¿Y ahora los tendría?

$\mathrm{Y}$ en comparación con la entrevista que ha hecho tu compañero/a de clase. ¿Crees que hay diferencias?

¿Cuáles? ¿Y ahora las hay?

¿Y hay similitudes? ¿Cuáles?

\section{Preguntas finales/cierre}

\author{
¿Hubieras cambiado algo de la entrevista? \\ ¿Qué opinas del resultado del proyecto? \\ Si tuvieras que señalar dos o tres cosas importantes que hayas aprendido ¿Cuáles serían? ¿Y dos o tres \\ cosas que ya sabías?
}

\section{Fuente: Elaboración propia}

Por último, en lo que respecta al segundo objetivo específico de este estudio -el fomento de las propuestas didácticas interdisciplinares que vinculan la disciplina histórica con la lingüística- los resultados que reflejan los participantes son prometedores tanto desde la perspectiva de los alumnos como desde el punto de vista de la profesora. En las entrevistas a los primeros se observa cómo en la práctica la mayor parte de los alumnos (sujetos 1, 2, 3,4, 8 y 9) integran los elementos de las distintas disciplinas que forman el módulo común. Así, por lo general, no separan elementos de la entrevista -propios de la disciplina lingüística- de cuestiones históricas desarrolladas en esta propuesta didáctica concreta. Estos alumnos señalan, por ejemplo, características de la entrevista observadas en el aula e impartidas por la profesora -como su "adaptabilidad al momento en el que se desarrolla la fuente”-, y las vinculan a elementos históricos del pasado. Muestran así una integración amplia de elementos de una y otra disciplina sin necesidad de que esta vinculación sea señalada directamente. Más aún, cabe señalar que las respuestas de los alumnos participantes evidencian que este tipo de propuestas didácticas, a pesar de ser interdisciplinares, no quedan en el recuerdo de los discentes como actividades interdisciplinares. Al respecto, únicamente el sujeto 2 se refirió directamente a esta actividad como una propuesta didáctica que une varias disciplinas. Para el resto de los alumnos, las actividades de esta propuesta son parte de la asignatura de historia.

Este hecho resulta ciertamente positivo dada la vinculación que muchos alumnos participantes hacen entre actividad o proyectos interdisciplinares y esfuerzo. Así lo refleja la profesora, evidentemente consciente de esta interdisciplinaridad, al señalar que el hecho de no hacerlo evidente al alumnado es "algo positivo, ya que si les digo que estamos haciendo dos asignaturas no les motiva, sino que les desanima porque creen que trabajaremos mucho más”. Al mismo tiempo, en su entrevista, la profesora señala la utilidad indiscutible de estos planteamientos 
interdisciplinares que permiten "alcanzar contenidos del libro y del currículum que nunca había podido dar”. Por ejemplo, señala la docente que los alumnos han sido capaces de "diferenciar entre fuentes primarias y secundarias, escritas y orales" de "señalar algunas características de las entrevistas, pero al hacerlas a sus familias las unían a cuestiones del pasado, a sus orígenes y contextos históricos”.

TABLA 5. Plantilla de observación

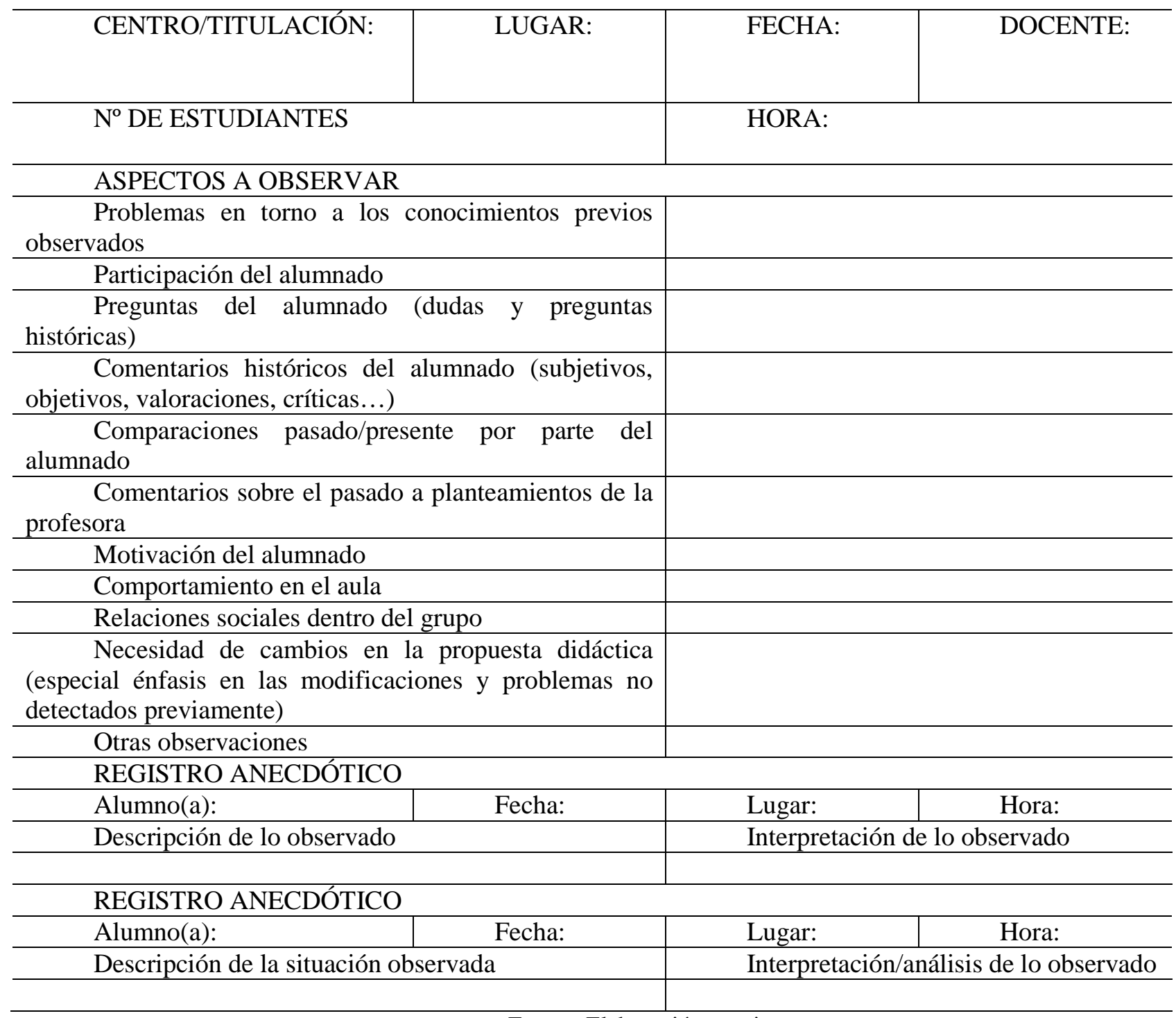

Fuente: Elaboración propia

\section{CONCLUSIONES}

En respuesta a las preguntas de investigación planteadas cabe señalar las siguientes reflexiones. En cuanto al segundo objetivo específico, el desarrollo de propuestas didácticas interdisciplinares y su utilidad en los módulos comunes de la FPB, los resultados muestran que éstas permiten vincular elementos relacionados y que favorecen un aprendizaje significativo y de mayor profundidad. Al mismo tiempo, observando la entrevista e informaciones obtenidas de parte de la docente, estas propuestas permiten de un lado, afrontar de forma exitosa un currículum que de otra manera se presentaba como inabarcable en la cronología del ciclo e inalcanzable dados los conocimientos previos del alumnado (Bello Muñoz y Carabantes de las Heras, 2015, p. 70), y de 
otro lado, evitar problemáticas típicas del alumnado de estos ciclos formativos tales como su desmotivación, falta de madurez, limitada resistencia a la frustración y escasa capacidad de trabajo en grupo (Fernández-García, García Llamas, y García Pérez, 2019; Moneo-Marín, Jiménez-Pérez, y Jiménez-Palacios, 2017).

En respuesta al primero de los objetivos específicos, la hipótesis de que la vinculación entre la disciplina histórica y las vivencias de los alumnos y de sus familias podía fomentar la motivación de los discentes, esta propuesta muestra su gran eficacia. Se convierte así en un planteamiento de gran utilidad debido a dos elementos fundamentales. El primero, su eficacia frente a un alumnado de FPB claramente desmotivado en los módulos comunes. En segundo lugar, su gran flexibilidad. Así, mientras esta vinculación de la historia con el contexto del discente se ha mostrado exitosa al desarrollarse en otros ciclos a partir del patrimonio material (Rivero y Pelegrín, 2015, p. 20), esta propuesta vincula historia y alumno a través de las emociones y de su pasado familiar, de un patrimonio inmaterial que nos rodea a todos en todos los lugares y del que todos somos partícipes. En definitiva, una vinculación que de esta manera no depende de la riqueza patrimonial de los contextos escolares y de la que todos los alumnos pueden ser partícipes por igual.

Finalmente, en respuesta al objetivo principal, esto es, el desarrollo de la empatía histórica para construir un pensamiento histórico crítico-creativo y desarrollar las competencias históricas, la propuesta didáctica muestra su eficacia y versatilidad. No solo se muestra el desarrollo del pensamiento histórico en el alumnado a partir de la empatía histórica -aunque con diferentes niveles entre los distintos discentes- sino que además, en la línea de análisis como los de GonzálezGonzález, Álvarez-Castillo, y Fernández-Caminero (2015). se puede observar la aparición de una empatía cultural vinculada al desarrollo de la empatía, el pensamiento y las competencias históricas.

Finalmente, cabe plantearse varias reflexiones que no son más que meras miradas hacia el futuro, pero que no por ello carecen de importancia. En primer lugar, si bien este estudio de caso es un breve acercamiento a una realidad compleja y amplia, presenta unos análisis cualitativos que permiten observar en profundidad realidades de suma relevancia. Más aún, describen algunas dinámicas que otros análisis ya han adelantado (Moneo-Marín, Jiménez-Pérez, y Jiménez-Palacios, 2017). Por ello, permite hipotetizar las posibilidades de extrapolación de este tipo de propuestas, al mismo tiempo que posibilita análisis en profundidad del impacto de estas necesarias acciones didácticas. En segundo lugar, y a tenor de la reflexión previa, tanto la plantilla de observación, como la propuesta y las entrevistas mostraron la importancia de la capacidad de adaptación en este tipo de propuestas didácticas para la FPB. Creemos, como ya señalaran Amores Fernánndez y Ritacco Real (2015, p. 117), que es esta flexibilidad la que termina permitiendo desarrollarlas con éxito. Sin embargo, paradójicamente, el currículum de estos mismos ciclos limita enormemente su flexibilidad y adaptabilidad, perjudicando directamente la práctica real en el aula. Y en tercer lugar, se ha de tomar en cuenta la conveniencia y necesidad de plantear proyectos y propuestas didácticas de investigación-acción que nos permitan reflexionar y analizar el día a día de las aulas de FPB y de sus módulos comunes. Su dificultad y escasez no puede limitar nuestra tarea y campo de reflexión. Más aún, los análisis de estos ciclos son a día de hoy todavía más necesarios si tenemos en cuenta las posibles modificaciones legislativas del futuro cercano y en ellas queremos evitar errores previos (García Gómez, 2014, p. 186).

\section{Referencias}

Alemán Falcón, J., Calcines Piñero, M. y Santana Hernández, R. (2017). La prevención del abandono escolar temprano a través de la aplicación de medidas en la formación profesional básica. En A. Rodríguez-Martín, Prácticas Innovadoras inclusivas: retos y oportunidades (pp. 411-419). Oviedo: Universidad de Oviedo. 
Almansa Pérez, R. (2018). La empatía como método humanístico de docencia de la historia: sugerencias didácticas en un panorama de desvalorización de los estudios históricos. Enseñanza de las ciencias sociales, 17, 87-98. DOI: 10.1344/ECCSS2018.17.8

Amores Fernández, J. y Ritacco Real, M. (2015). De los Programas de Cualificación Profesional Inicial (PCPI) como medida de prevención del fracaso escolar a la Formación Profesional Básica. Un estudio sobre el éxito y fracaso de alumnos en riesgo de exclusión educativa en Educación Secundaria. Revista de Investigación en Educación, 13(1), 105-120.

Bello Muñoz, M. y Carabantes de las Heras, I. (2015). Una formación Profesional Básica que ni es básica ni es profesional. Fórum Aragón, 15, 68-70.

Burke, P. (2005). Visto y no visto: el uso de la imagen como documento histórico. Barcelona: Crítica.

Butrus, N. y Witenberg, R. (2013). Some Personality Predictors of Tolerance to Human Diversity: The Roles of Openness, Agreeableness, and Empathy. Australian Psychologist, 48, 290-298. DOI: $10.1111 / \mathrm{j} .1742-9544.2012 .00081 . \mathrm{x}$

Carr, W. y Kemmis, S. (1988). Teoría crítica de la enseñanza. La investigación-acción en la formación del profesorado. Barcelona: Martínez Roca.

Cejudo Cortés, C. A., y Corchuelo Fernández, C. (2018). La formación Profesional Básica como medida socioeducativa para prevenir el abandono escolar prematuro. En-Clave Pedagógica, 14, 61-66.

Cid-Sillero, S., Santiago-Ramajo, S. y Martín-Lobo, M. (2018). Relación entre las fucnioens ejecutivas y la empatía y su influencia en el rendimiento académico en alumnado de Formación Profesional Básica. Electronic Jorunal of Research in Educational Psychology, 16(2), 517-536. DOI: 10.25115/ejrep.v16i46.2233

Corbacho Casas, F. y Pons García, J. (2006). Jóvenes en desventaja y cohesión social. Educación y futuro para todos. Revista de Educacion, 341, 237-258.

Cutanda López, M. T. (2014). De los Programas de Cualificación Profesional Inicial (PCPI) a la Formación Profesional Básica: algunas consideraciones. Revista Educativa Hekademos, 16, Año VII, 69-79.

Davis, M. (1983). Measuring individual differences in empathy: Evidence for a multidimensional approach. Journal of Personality, 51, 113-126.

Díaz Barriga, F. (1998). Una aportación a la didáctica de la historia. La enseñanza aprendizaje de habilidades cognitivas en el bachillerato. Perfiles Educativos, 82.

Domínguez, J. (2015). Pensamiento histórico y evaluación de competencias. Barcelona: Ed. Graó.

Doñate Campos, O. y Ferrete Sarria, C. (2019). Vivir la historia: Posibilidades de la empatía histórica para motivar al alumnado y lograr una comprensión afectiva de los hechos históricos. Didáctica de las Ciencias Experimentales y Sociales, 36, 47-60. DOI: $10.7203 /$ DCES.36.12993

Eisenberg, N. (2000). Emotion, Regulation, and Moral Development. Annual Review of Psychology, 51, 665-697. DOI: 10.1146/annurev.psych.51.1.665

Fernández-García, A., García Llamas, J. y García Pérez, M. (2019). La formación profesional básica, una alternativa para atender las necesidades educativas de los jóvenes en riesgos social. Revista de Humanidades, 36, 211-232.

García Gómez, S. (2014). La investigación sobre el alumnado de los Programas de Cualificación Profesional Inicial ¿¿Punto de partida de la Formación Profesional Básica? Revista de Investigación en Educación, 12(2), 176-190.

González González, M. y Moreno Yus, M. (2013). El Programa de Cualificación Profesional Inicial: Entre la integración o la marginación en los contextos organizativos donde se desarrolla. Revista de Investigación en Educación, 11(1), 118-133.

González, N., Henríquez, R., Pagès, J. y Santiesteban, A. (2009). El aprendizaje de la empatía histórica (eh) en educación secundaria. Análisis y proyecciones de una investigación sobre la 
enseñanza y el aprendizaje del conflicto y la convivencia en la Edad Media. En R. M. Ávila, B. Borghi y I. Mattozzi, L'educazione alla cittadinanza europea e la formazione degli insegnanti. Un progetto educativo per la "Strategia di Lsibona": atti XX Simposio Internacional de Didáctica de las Ciencias Sociales (pp. 283-290). Bologna: Asociación Universitaria de Profesorado de Didáctica de las Ciencias Sociales.

González-González, H., Álvarez-Castillo, J.-L. y Fernández-Caminero, G. (2015). Desarrollo y validación de una escala de medida de la empatía intercultural. RELIEVE, 21(2), 1-18. DOI: $\underline{10.7203 / \text { relieve.21.1.7841 }}$

Latorre, A. (2003). La investigación-acción. Conocer y cambiar la práctica educativa. Barcelona: Editorial Graó.

Lee, P., y Shemilt, D. (2009). Is any explanation better than none? Teaching History, 137, 42-49.

Lee, P. y Shemilt, D. (2011). The concept that Dares not speak its name: should empathy come out of the closet? Teaching History, 143, 39-49.

Leoné Puncel, S. y Mendiola Gonzalo, F. (2007). Voces e imágenes en la historia: fuentes orales y visuales: investigación histórica y renovación pedagógica. Pamplona: Universidad Pública de Navarra.

Martín Ayasta, D. (2010). La empatía en la enseñanza de la historia. A la búsqueda de la empatía en un aula de los Andes. Iber: Didáctica de las Ciencias Sociales, Geografía e Historia, 64, 102110.

Molina Puche, S. y Egea Zapata, J. (2018). Evaluar la adquisición de las competencias históricas en Secundaria: un estudio de caso centrado en la empatía histórica. ENSAYOS, Revista de la Factulad de Educación de Albacete, 33(1), 1-22.

Moneo-Marín, Á., Jiménez-Pérez, R. y Jiménez-Palacios, R. (2017). Evolución de la madurez emocional en alumnos de Formación Profesional Básica mediante el empleo de actividades fuera del contexto escolar en el área de ciencias naturales. Revista Eureka sobre Enseñanzas y Divulgación de las Cienicas, 14(1), 69-85.

Montserrat, C. y Melendro, M. (2017). ¿Qué habilidades y competencias se valoran de los profesionales que trabajan con adolescencia en riesgo de exclusión social? Análisis desde la acción socioeducativa. Educación XX1, 20(2), 113-135. DOI: 10.5944/educxx1.19034

Moreno, G., España, E., y Blanco, Á. (2016). Propuesta didáctica sobre la compra de un coche para trabajar competencias clave en la Educación Secundaria. Revista Eureka sobre Enseñanza y Divulgación de las Ciencias, 13(3), 604-616.

Oliva Martínez, J. (2012). Dificultades para la implicación del profesorado de Secundaria en la lectura, innovación e investigación en didáctica de las ciencias (II): el problema del "manos a la obra". Revista Eureka sobre Enseñanza y Divulgación de las Ciencias, 9(2), 241-251.

Olmos Rueda, P. y Mas Torelló, O. (2013). Youth Academic Failure and second chance training programmes. REOP Revista Española de Orientación y Psixopedagogía, 24(1), 78-93.

Ortiz, M. y Borjas, B. (2008). La Investigación Acción Participativa: aporte de Fals Borda a la educación popular. Espacio Abierto, 17(4), 615-627.

Perarnau, E. y Vieyra, E. (2017). Memoria, imaginación histórica y empatía histórica: dos experiencias con adultos mayores. Argonautas, 7(9), 1-8.

Ríos Rincón, S. M. (2017). Formación de la empatía a través del uso de la imagen artística. El caso de las víctimas de la violencia en Colombia. (pensamiento), (palabra). Y Obra, 18(18), 53-63.

Rivero, P. y Pelegrín, J. (2015). Aprender historia desde la empatía experiencias en Aragón. Aula $240,18-22$.

Rodríguez Sabiote, C., Álvarez Rodríguez, J. y Moreno Yus, M. (2009). Los programas extraordinarios de atención a la diversidad: implicaciones psicológicas, académico-cognitivas y sociales en el alumnado de la Comunidad Autónoma de Andalucía. Profesorado. Revista de Currícuclum y Formación del Profesorado, 13(3), 175-192. 
Ryan, G. y Bernard, H. (2003). Data Management and Analysis Methods. En N. Denzin y Y. Lincoln, Handbook of Qualitative Research (pp. 769-802). Thousand Oaks: Sage Puclications.

Salguero, J., Fernández-Berrocal, P., Ruiz-Aranda, D., Castillo, R. y Palomera, R. (2011). Inteligencia emocional y ajuste psicosocial en la adolescencia: El papel de la percepción emocional. European Journal of Education and Psychology, 4(2), 143-152.

Salmón Perrilliat, E. (2017). La empatía como metodología. Una receta contra el exotismo. Relaciones Estudios de Historia y Sociedad, 152, 367-378.

Santisteban Fernández, A. (2010). La formación de competencias de pensamiento histórico. Clío y Asociados, 14, 34-56.

Seixas, P. y Morton, T. (2013). The Big Six Historical Thinking Concepts. Toronto: Nelson College. Shemilt, D. (1984). Beauty and the Philosopher: Empahty in History and Classroom. En A. Dickinson, J. Lee (eds.), Learning History (pp. 39-84). Londres: Heineman Educational Books.

Tarabini, A. y Montes, A. (2015). La agenda política contra el abadono escolar prematuro en España: la LOMCE contra las evidenias internacionales. Revista de la Asociación de Inspectores de Educación de España, 23, 1-20.

Trepat, C.-A. (1995). Procedimientos en historia: un punto de vista didáctico. Barcelona: ICE/UB.

\section{CÓMO CITAR ESTE ARTÍCULO}

Ciriza-Mendívil, C. D. (2021). La empatía histórica, una propuesta didáctica clave para la Formación Profesional Básica. Didáctica de las ciencias experimentales y sociales, 40, 51-66. DOI: 10.7203/DCES.40.17309 
